\title{
A PESQUISA DE IMPACTO ECONÔMICO COMO SUBSÍDIO PARA AS TOMADAS DE DECISÃO EM GESTÃO DE EVENTOS ESPORTIVOS: ESTUDO DE CASO DO X GAMES BRASIL 2013
}

\author{
ECONOMIC IMPACT RESEARCH INFORMING DECISION-MAKING IN MANAGEMENT \\ OF SPORTS EVENTS: A CASE STUDY ON THE X GAMES BRAZIL 2013
}

LA INVESTIGACIÓN DEL IMPACTO ECONÓMICO COMO SUBSIDIO PARA LA TOMA

DE DECISIÓN EN GESTIÓN DE EVENTOS DEPORTIVOS: ESTUDIO DE CASO DEL

X GAMES BRASIL 2013

\section{Geraldo Ricardo Hruschka Campestrini, Lamartine DaCosta*}

Palavras chave:

Economia.

Impacto primário

Esportes.

Gestão de recursos.
Resumo: O objetivo deste estudo foi analisar o impacto econômico de eventos esportivos sobre uma localidade, com efeitos sobre as tomadas de decisão em gestão. 0 estudo utilizou dados do X Games Brasil, realizado em Foz do Iguaçu/PR em 2013, quando se realizou uma pesquisa de campo quantitativa, com a aplicação de questionário no local do evento, somada à análise documental da organização do evento, compondo uma análise sobre o seu custo-benefício. $O$ impacto econômico calculado foi de $\mathrm{R} \$ 51,7$ milhões. 0 ponto central de reflexão passa pela compreensão de como essas informações econômicas e financeiras implicam tomadas de decisão em gestão de eventos esportivos, uma vez que existem pontos de convergência entre as parcerias público-privadas comuns neste tipo de acontecimento. Sugere-se, assim, um modelo analítico mais racional para corroborar 0 aporte público em eventos esportivos.
Keywords:

Economics.

Primary impact.

Sports.

Resource

management.
Abstract: The aim of this study was to analyze the economic impact of sports events on places, with consequences for decision-making in management. The study used data from the X Games Brazil held in Foz do Iguaçu, PR, in 2013. A quantitative field study was conducted at the event as well as a document analysis of its organization - both making up a cost-benefit analysis. The estimated economic impact was estimated as BRL 51.7 million. The discussion focuses on understanding how that economic and financial information reflects on decision-making in sports event management as there are points of convergence between public-private partnerships that are common in such events. Therefore, a more rational analytical model is suggested to support state contribution to sports events.
Palabras clave: Economía. Impacto primario. Deportes. Gestión de recursos.
Resumen: El objetivo de este estudio fue analizar el impacto económico de los eventos deportivos en una localización, con efectos sobre la toma de decisiones en gestión. El estudio utilizó datos de los X Games de Brasil, realizado en Foz do Iguaçu/PR en 2013. Se realizó, entonces, una investigación cuantitativa de campo con un cuestionario aplicado en el local del evento, además del análisis documental de la organización del evento, componiendo un análisis de la relación costo beneficio. El impacto económico estimado fue de $\mathrm{R} \$ 51,7$ millones. El punto central de la reflexión es la comprensión de cómo esas informaciones económicas y financieras redundan en la toma de decisiones en gestión de eventos deportivos, ya que existen puntos de convergencia entre las colaboraciones público-privadas comunes en este tipo de actividad. Se sugiere, por lo tanto, un modelo analítico más racional para corroborar el aporte público en eventos deportivos.
*Universidade do Estado do Rio de Janeiro. Rio de Janeiro, RJ, Brasil. E-mail: geraldocamper@terra.com.br

Recebido em: 26-08-2016 Aprovado em: 27-01-2017 


\section{INTRODUÇÃOO}

A análise de eventos esportivos enseja um debate amplo sobre a sua viabilidade econômica à luz dos gastos públicos e privados realizados, conquistando importância significativa nos últimos anos. Contudo, boa parte dos estudos de impacto econômico atende simplesmente objetivos específicos, apresentando dificuldade para legitimar as tomadas de decisão de todo setor produtivo. Visando ao aperfeiçoamento da gestão de eventos esportivos, é necessário que as informações econômicas sejam mais simples, qualificadas e reais.

Para que isso ocorra, estudos dessa natureza não devem se restringir a informações econômicas, apesar de fazerem uso de dados e índices econométricos para a sua sustentação. Propõe-se integrar uma visão ampla para a aplicabilidade no contexto da gestão de pequenos, médios e grandes eventos, contribuindo com um processo de racionalização dos investimentos. O objetivo deste estudo é apresentar uma metodologia para verificar o impacto econômico de eventos esportivos a partir dos dados coletados no X Games Brasil, evento realizado em 2013 em Foz do Iguaçu, Paraná. Espera-se que os resultados encaminhem reflexões sobre os processos de tomadas de decisão sobre investimentos públicos em eventos esportivos.

Os principais pontos de inflexão dos estudos de impacto econômico guardam relação com o debate sobre o papel do poder público no apoio e financiamento desses eventos. Neste sentido, a abordagem comumente passa pelas análises macroeconômicas, microeconômicas e pelos aspectos intangíveis.

A verificação de dados macroeconômicos dos países ou cidades-sede de megaeventos aponta para resultados inconsistentes sobre os reais benefícios ante o elevado investimento que é comumente realizado pelo poder público (BRUNET; XINWEN, 2009; PORTER; FLETCHER, 2008; TIEN; LO; LIN, 2011; YU; LIU, 2011). O saldo costuma ser negativo pelos altos custos de investimento em infraestrutura (BRUNET; XINWEN, 2009; ACCESS ECONOMIC PTY LIMITED, 2010).

Outros estudos buscaram amparo na análise do impacto intangível para se justificar os investimentos realizados (WICKER; HALLMANN et al., 2012). Destaque-se 0 orgulho da comunidade, fortalecimento da identidade e notoriedade da marca (HUANG; HANQI, 2011; BARGET; GOUGOUT, 2010; GRATTON; PREUSS, 2008; LEE, 2014), participação esportiva (FRAWLEY; CUSH, 2011; KAVETSOS; SZYMANSKI, 2009; BARGET; GOUGOUT, 2010), posicionamento político e social (YU; LIU, 2011), evocação do jogo limpo, da paz e da igualdade (PREUSS; SCHÜTTE, et al., 2014) e a aceleração para grandes transformações urbanas nas cidades (SILVESTRE, 2008).

Uma terceira corrente de análises procura entender os aspectos microeconômicos. Assim, separa os eventos esportivos de pequeno, médio e grande porte dos megaeventos, que exigem grandes transformações urbanas e estruturais, como a construção de instalações em um nível de excelência que atenda uma demanda pontual. Eventos menores apresentam necessidades operacionais menos dispendiosas, aproveitando a já existente infraestrutura ou mesmo utilizando estruturas temporárias, sinalizando resultados econômicos mais positivos (BARGET; FERRAND, 2012; BERNTHAL; REGAN, 2004; CROMPTON, 1999; DANIELS; NORMAN, 2003; RAMCHANDANI; COLEMAN, 2012). Isso ocorre pois tais eventos operam com baixo custo de oportunidade e com os recursos da própria comunidade (WALO; BULL; BREEN, 1996; GRATTON; SHIBLI; COLEMAN, 2006), sendo mais factível a mensuração 
dos benefícios econômicos gerados (CROMPTON, 1995). Os maiores benefícios são o custo reduzido de candidatura, o uso de infraestrutura já existente, os gastos públicos diminutos ou inexistentes e o impacto pequeno na comunidade local (DANIELS; NORMAN, 2003).

O impacto microeconômico é comumente calculado a partir de análise de custobenefício, que é considerada a forma mais adequada para avaliar o investimento público em projetos desta natureza (JEANRENAUD, 2006). Os estudos medem os impactos diretos, que são os gastos brutos dos visitantes e/ou da organização do evento, o impacto indireto, compreendido como a recirculação do dinheiro após o primeiro gasto, e o impacto induzido, que representa o gasto adicional a partir do ganho salarial gerado pelo evento em favor dos colaboradores (CROMPTON, 1995).

$\mathrm{Na}$ análise, é preciso ter cuidado com os efeitos multiplicadores, que tendem a inflar os resultados (JEANRENAUD, 2006), passando uma imagem inverídica sobre o fato e, também, relativizar as características locais que, ao atraírem um evento esportivo, cumprem ou complementam um objetivo específico (SACK; JOHNSON, 1996). Assim, é importante a análise da matriz econômica de cada localidade, que pode impactar de distintas maneiras em função das suas particularidades (CROMPTON, 1995), como a existência ou não de hotéis comerciais (WILSON, 2006), o fato de ser uma área rural ou urbana ou de ter maior ou menor oportunidade de entretenimento (BERNTHAL; REGAN, 2004). Esse conjunto de elementos interfere no comportamento de consumo dos visitantes e na participação da comunidade local.

Pelo exposto, nota-se que o estudo do impacto econômico de eventos esportivos não pode ser isolado e dissociado de um conjunto de elementos que, agrupados, refletem sobre a sua gestão em termos de parcerias e construção de cenários.

\section{DECISÕES METODOLÓGICAS}

A pesquisa seguiu critérios de análise do custo-benefício, baseado nas entradas e saídas de recursos na cidade-sede do evento esportivo para o cálculo do seu impacto econômico. Estudos de custo-benefício buscam identificar os gastos da organização do evento e seu respectivo impacto no local somado à aplicação de questionário in loco com o público presente para mapear o comportamento de consumo na região. Esses parecem ser o melhor meio para se estimar as consequências econômicas de um evento, uma vez que admitem efeitos positivos e negativos, o que significa que poderão apontar resultados desfavoráveis no término do processo (JEANRENAUD, 2006; TAKS; KESENNE et al., 2011), pois têm em conta as economias de escala, a produção próxima ao limite da sua capacidade e as adaptações de preços (KASIMATI, 2003).

A coleta de informações foi realizada em dois momentos distintos e consecutivos. 0 primeiro na pesquisa de campo, realizada com quatro pesquisadores, que fizeram abordagem pessoal direta, aplicando questionário físico a uma amostra aleatória simples (GIL, 2010) aos espectadores presentes no evento X Games Brasil entre os dias 18 e 21 de abril de 2013, realizado em Foz do Iguaçu/PR. Os participantes, além de preencherem o questionário (OBSERVATÓRIO DO TURISMO DA CIDADE DE SÃO PAULO, 2012), assinavam termo de livre consentimento, em que se previa que "[...] todas as informações seriam tratadas de forma sigilosa, sem a identificação individual dos respondentes". Ao todo, foram realizadas 1.182 aplicações do questionário, sendo que 1.113 respostas foram consideradas válidas, dado que 
se descartaram questionários incompletos ou mal preenchidos. A amostra representou um universo de 27.093 pessoas presentes nos quatro dias de evento, o que representa margem de erro de $3 \%$ e nível de confiança de $98 \%$.

O segundo momento levou em conta os dispêndios relativos à organização e gestão do evento, considerando os montantes investidos pelos entes públicos e privados, bem como 0 consumo impactado no local. Tais informações foram fornecidas oficialmente pela organizadora do evento, a empresa BSB Marketing Esportivo S/A, pela detentora dos direitos do evento, a ESPN, do Grupo The Walt Disney Company por meio de planilhas e relatórios internos, e pela Prefeitura Municipal de Foz do Iguaçu. Essas informações se caracterizam como uma coleta de estudo de caso, uma vez que envolvem a apresentação e a análise de informações detalhadas de um ou mais assuntos em relação a um evento ou vida particular (EDWARDS; SKINNER, 2009).

A composição desses dois vetores permitiu construir uma visão ampla sobre o evento, o que repercute nos debates sobre a gestão e as tomadas de decisão em esporte.

\section{ANÁLISE}

Um dos princípios-base da análise foi o de procurar verificar o ganho incremental sobre a realização do evento e não somente o ganho bruto (WEINSTEIN; DEGRACIA; LIN, 2010). $\mathrm{O}$ cruzamento de dados se deu pelo histórico de comportamento do turista em Foz do Iguaçu do Paraná (FDIPR) quanto às despesas com hospedagem, transporte, alimentação, compras e entretenimento, comparado com os dados informados pelos turistas do evento em análise.

Como FDIPR é uma cidade eminentemente turística, que está entre os três principais destinos turísticos do Brasil (BRASIL, 2014), não parece fazer sentido analisar os valores brutos, uma vez que se nota um fluxo de turistas relativamente ótimo ao longo do ano e, por isso, a realização de eventos esportivos contribuem apenas para incrementar o turismo em um curto espaço de tempo. Não há mudança significativa na cidade para fazer aquilo que ela já está vocacionada a realizar normalmente.

\subsection{Resultado do impacto do turismo}

Ao todo, foram vendidos 58.250 ingressos para os quatro dias de evento. De acordo com as respostas, os entrevistados visitaram o evento por 2,15 dias em média, o que sugere um total de 27.093 visitantes únicos.

Deste total, 59,7\% são turistas que pernoitaram pelo menos um dia em FDIPR. 30,2\% residem em FDIPR e os demais 10,1\% são representados por visitantes de cidades vizinhas que não pernoitaram em FDIPR, tendo um comportamento similar ao residente local.

Para efeitos de cálculo do impacto econômico dos turistas, considerou-se tão somente o contingente de $94 \%$ dos respondentes que declararam ter viajado para FDIPR por conta do X Games, representando um universo de 15.202 turistas. Desconsideraram-se aqueles visitantes que viriam para a cidade de qualquer maneira, mais cedo ou mais tarde, e somente combinaram a ocorrência do evento para reforçar sua agenda (ACCESS ECONOMIC PTY LIMITED, 2010).

A população considerada foi identificada pelo seguinte cálculo: 
$A=\left(\frac{1}{\text { Vist }}\right) \times T \times X G$

Em que:

A = População Considerada para os Cálculos de Impacto Econômico

I = Quantidade de Ingressos Vendidos

Vist = Média de Ida ao Local do Evento durante a permanência em FDIPR

$T=$ Quantidade Relativa de Turistas

$X G=$ Quantidade Relativa de Turistas que declararam ida à cidade em razão do evento

O gasto médio destes turistas em FDIPR ao longo da sua permanência foi de R \$ $1.073,01$, sendo que o período de permanência médio foi de 2,63 dias por pessoa. Os grupos, em média, foram formados por 1,85 pessoas, considerando homens, mulheres e crianças. 0 intervalo de confiança em relação aos gastos médios por pessoa está representado na Figura 1.

Figura 1 - Intervalo de Confiança sobre a média de gastos dos turistas em Foz do Iguaçu durante a sua permanência na cidade para o evento X Games.

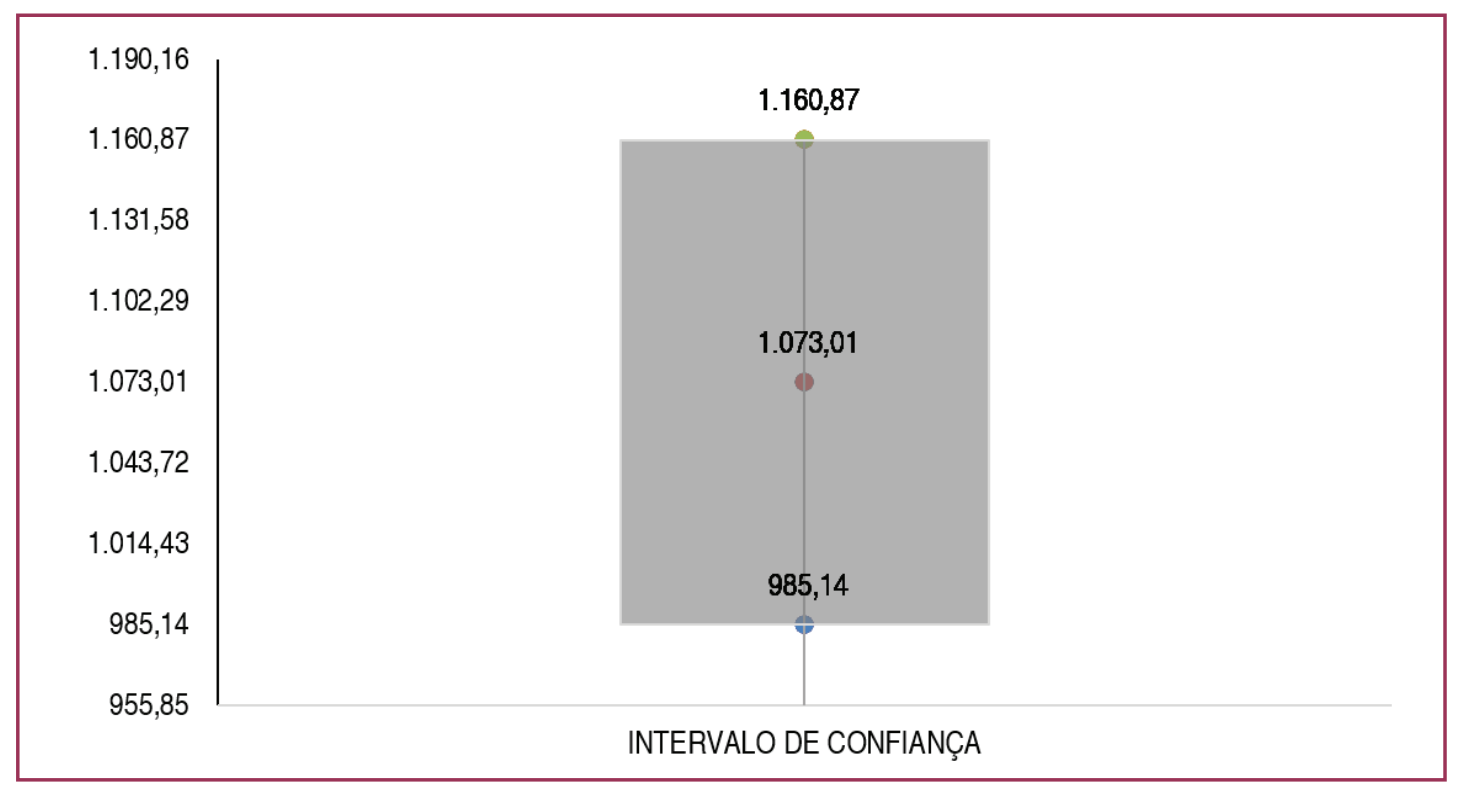

Fonte: da pesquisa.

Proporcionalmente, os maiores dispêndios dos turistas são canalizados para a hospedagem, que consome quase 1/4 deste total. Em segundo lugar, aparece o item compras, com $21,6 \%$ do total e em terceiro lugar o item lazer/entretenimento, conforme Tabela 1.

Tabela 1 - Participação relativa dos gastos pessoais dos turistas em Foz do Iguaçu durante a sua permanência na cidade para o evento X Games.

\begin{tabular}{cc}
\hline TIPO DE GASTO (R\$) & PARTICIPAÇÃO PER CAPITA TOTAL \\
\hline HOSPEDAGEM & $24,1 \%$ \\
COMPRAS NA REGIÃO & $21,6 \%$ \\
LAZER/ENTRETENIMENTO & $17,3 \%$ \\
COMPRAS EM FOZ DO IGUAÇU & $15,6 \%$ \\
ALIMENTAÇÃO & $15,5 \%$ \\
TÁXI/TRANSPORTE COLETIVO & $5,9 \%$ \\
\hline
\end{tabular}


No item "compras" se evidencia uma das características locais que tendem a direcionar o comportamento do turista. Como FDIPR está em uma região de fronteira com o Paraguai, que é um país com regime de tributação que favorece a compra de produtos importados, há uma tendência do público visitante em reservar algum investimento para, na oportunidade de estar em FDIPR, efetuar compras na região. É o que se identifica na pesquisa. Daí a necessidade em se retirar do cálculo de impacto econômico em prol de FDIPR os 21,6\% de gasto do público com "Compras na Região", uma vez que este valor não ficou retido em benefícios diretos para a cidade.

Assim, para não olhar de forma míope os números da pesquisa, deve-se considerar a realidade local, em que se observa que:

1) A média de permanência do turista está registrada em 2,28 dias e a taxa média de ocupação hoteleira no mês de abril de 2013 esteve registrada em 53,3\% da rede, que é composta por 26.368 leitos (OBSERVATÓRIO DE TURISMO DE FOZ DO IGUAÇU, 2014c; OBSERVATÓRIO DE TURISMO DE FOZ DO IGUAÇU, 2014a).

2) $23 \%$ dos respondentes indicaram meio de hospedagem não relacionado com os meios convencionais. Assim, é possível afirmar que 10.290 pessoas ocuparam a rede hoteleira da cidade, acrescentando 39\% à ocupação hoteleira naquele período de evento.

3) Não se registrou queda no fluxo de turistas regulares em aeroportos ou visitas a parques da cidade durante o evento (BRASIL. MINISTÉRIO DO TURISMO, 2014; FOZ DO IGUAÇU. SECRETARIA MUNICIPAL DE TURISMO, 2014). Estes últimos se caracterizam tradicionalmente como locais-alvo para os visitantes de FDIPR. Esta constatação faz com que o gasto médio do turista que foi ao $X$ Games seja totalmente creditado como impacto econômico em favor da cidade.

4) Os turistas regulares somados aos turistas do $X$ Games proporcionaram uma ocupação hoteleira média de $92,3 \%$ no período do evento.

5) O gasto médio do turista convencional em FDIPR é de R $\$ 347,29$ (OBSERVATÓRIO DE TURISMO DE FOZ DO IGUAÇU, 2014b), ante os R\$ 813,17 identificados na pesquisa sobre os visitantes do X Games, após descontar-se o gasto com compras na região.

O resultado do impacto direto do X Games foi, portanto, calculado em $\mathrm{R} \$ 12.361 .810,34$, encontrado a partir da fórmula a seguir:

$I E T=((G M D T C \times Q C \times D C)-((T O H M-T O H E) \times G M D T R h \times D T R))$

Em que:

IET = Impacto Econômico do Turismo Correspondente do Evento Esportivo.

GMDTc = Gasto Médio Diário do Turista do Evento Esportivo, que ficou em Hospedagem Comercial, Per Capita, durante a sua Estadia na Localidade.

$\mathrm{QC}=$ Quantidade de Turistas do Evento Esportivo em Hospedagem Comercial.

DC = Quantidade Média de Dias de Permanência na Cidade-Sede do Evento Esportivo, do Turista do

Evento Esportivo em Hospedagem Comercial.

TOHM = Taxa de Ocupação em Hospedagem Comercial Média e Histórica com o Turista Regular no

Período do Evento Esportivo (últimos 3 anos).

TOHE = Taxa de Ocupação em Hospedagem Comercial durante o Evento Esportivo com o Turista Regular.

GMDTRh = Gasto Médio Diário, Per Capita, Histórico do Turista Regular com Hospedagem Comercial durante a sua Estadia na Cidade-Sede do Evento Esportivo.

DTR = Média Histórica de Dias de Permanência do Turista Regular na Cidade-Sede do Evento Esportivo. 
Uma vez que a média de ocupação hoteleira do turista regular foi considerada a mesma para o período do evento em análise, tem-se uma taxa de desconto equivalente a zero. Assim, o resultado bruto calculado para o impacto foi gerado a partir da base de gastos per capita do turista que foi ao evento e seus respectivos dispêndios em FDIPR. Para o modelo, a Figura 2 ilustra a lógica do cálculo proposto.

Figura 2 - Modelo geral desenvolvido para um cálculo mais racional do impacto econômico do turismo em um evento esportivo.

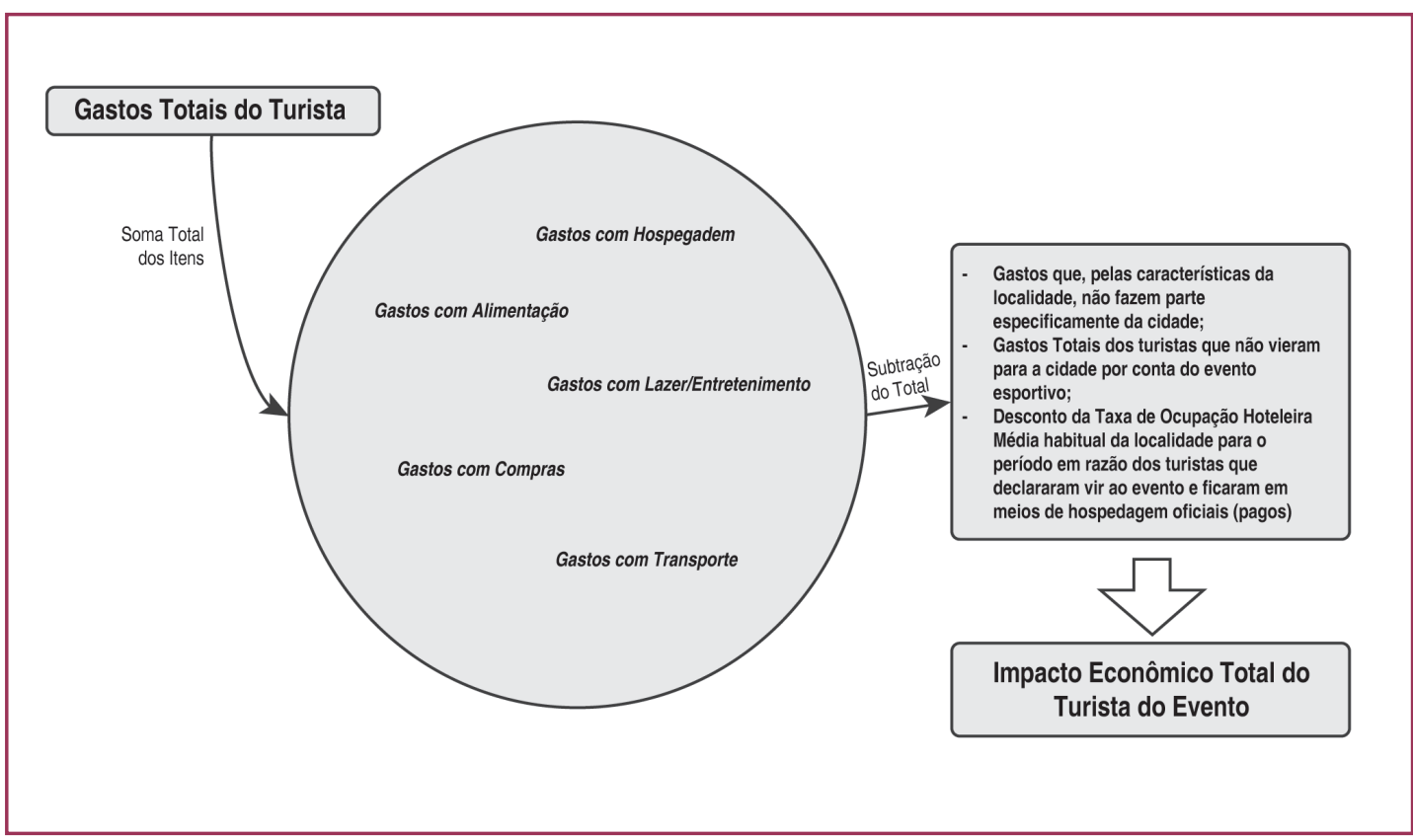

Fonte: da pesquisa.

\subsection{Resultado dos gastos com a gestão e produção do evento}

Este é um gasto que leva em conta as tipologias dos dispêndios e o local de realização e efetivação do pagamento. Conforme o tamanho do evento ante o tamanho da cidade que 0 recebe, é possível que nem todos os serviços e insumos sejam adquiridos na localidade.

É o caso do evento sob análise. FDIPR, por característica, não dispunha de todas as especificidades e necessidades para a operação e gestão que o evento exigia. Portanto, há que se considerar o gasto total como um impacto para a localidade em relação ao evento após serem descontadas eventuais contratações de serviços ou produtos de fora da cidade. A "importação" desses insumos não faz parte do cálculo do impacto econômico em favor da localidade.

A Tabela 2 mostra o gasto total por rubricas, em dólares e em reais, convertidos a preços do período de realização do evento ${ }^{1}$, com respectivos percentuais do total. A última coluna apresenta a proporção destes gastos realizados especificamente na cidade de FDIPR, que representa $28,3 \%$, ou $\mathrm{R} \$ 11.133 .106,46$ em favor da cidade. 
Tabela 2 - Gastos Totais e Efetivados em Foz do Iguaçu para a Realização do X Games.

\begin{tabular}{|c|c|c|c|c|c|}
\hline \multirow{2}{*}{ ITENS CONTRATADOS } & \multicolumn{3}{|c|}{ GASTOS TOTAIS DO EVENTO } & \multicolumn{2}{|c|}{$\begin{array}{c}\text { GASTOS REALIZADOS EM FOZ DO } \\
\text { IGUAÇU }\end{array}$} \\
\hline & US\$ & RS & PERCENTUAL\% & $\mathrm{R} \$$ & PERCENTUAL \% \\
\hline Serviços Médicos & 330.825 & 662.322 & 1,7 & $475.601,88$ & 71,8 \\
\hline Logística e Transporte & 1.705 .120 & 3.413 .704 & 8,7 & $249.260,33$ & 7,3 \\
\hline Produção e Operação & 10.602 .304 & 21.226 .149 & 53,9 & $4.104 .389,10$ & 19,3 \\
\hline Recursos Humanos & 1.888 .419 & 3.780 .675 & 9,6 & - & 0,0 \\
\hline Hospedagem & 320.241 & 641.133 & 1,6 & $641.133,28$ & 100,0 \\
\hline Catering e Hospitalidade & 861.449 & 1.724 .648 & 4,4 & $1.724 .648,17$ & 100,0 \\
\hline Tecnologia de Informação & 577.860 & 1.156 .895 & 2,9 & $774.580,81$ & 67,0 \\
\hline Marketing & 96.704 & 193.605 & 0,5 & $162.285,40$ & 83,8 \\
\hline Operações de TV & 1.899 .771 & 3.803 .401 & 9,7 & $1.746 .408,39$ & 45,9 \\
\hline Administrativo-Financeiro & 568.590 & 1.138 .336 & 2,9 & - & 0,0 \\
\hline Segurança & 692.496 & 1.386 .400 & 3,5 & $1.050 .951,87$ & 75,8 \\
\hline Outros & 119.340 & 238.923 & 0,6 & $203.847,24$ & 85,3 \\
\hline Total & 19.663 .120 & 39.366 .192 & 100,0 & 11.133 .106 & 28,3 \\
\hline
\end{tabular}

Fonte: documentos internos da organização do evento.

Curiosamente, a cidade de São Paulo/SP foi a que mais recebeu recursos provenientes dos X Games por parte da organização do evento, somando-se $\mathrm{R} \$ 18,3$ milhões. Este é mais um indicador importante ao se analisar o impacto econômico local para se perceber a capacidade que o município-sede do evento possui para suprir todas as demandas inerentes a ele.

\subsection{Impacto total}

O Impacto Total, bruto, é calculado pela soma do Impacto Econômico do Turismo (IET) com o Impacto Econômico da Gestão, Operação e Organização do Evento Esportivo (IETGOO), que totalizou $\mathrm{R} \$ 51.728 .002,00$. A partir deste valor bruto, é necessário relativizar a representatividade do impacto para cada ente público participante com investimentos no respectivo evento esportivo.

\subsection{Análise do investimento público}

Pela dimensão do X Games, houve um aporte e uma coparticipação de entes Federal, Estadual e Municipal para subsidiar a realização do evento. Do Ministério do Esporte houve um investimento de $\mathrm{R} \$ 5.834 .838,92$. Do Governo do Estado do Paraná, foram R $\$ 2.140 .000,00$. E da Prefeitura Municipal, $\mathrm{R} \$ 3.749 .437,30$. No total, foram $\mathrm{R} \$ 11.724 .276,22$ em recursos públicos aplicados ao evento.

Pelo levantamento dos números combinados entre os dispêndios do turista do X Games somados aos custos de produção e operação do evento e verificando-se os investimentos públicos realizados, apresenta-se a Tabela 3 , que demonstra a recuperação tributária² calculada sobre cada setor de atividade impactado.

2 Referenciada com base nas alíquotas de impostos e tributos de cada insumo realizado em prol do X Games. 
Tabela 3 - Síntese do Investimento dos Governos Federal, Estadual e Municipal para a realização do X Games em Foz do Iguaçu e a respectiva recuperação tributária de cada ente.

\begin{tabular}{cccc}
\hline ITENS (R\$) & FEDERAL & ESTADUAL & MUNICIPAL \\
\hline Investimento no X Games & $5.834 .838,92$ & $2.140 .000,00$ & $3.749 .437,30$ \\
Receita Tributária Realizada & $4.311 .367,61$ & $1.730 .975,63$ & $462.750,65$ \\
Superávit (Déficit) & $(1.523 .471,31)$ & $(409.024,37)$ & $(3.286 .686,65)$ \\
\hline
\end{tabular}

Fonte: da pesquisa.

\section{DISCUSSÃO}

O modelo de análise proposto passa pela verificação dos custos de gestão e produção do evento, sem considerar possíveis custos de construções ou reformas de instalações esportivas permanentes, somados às características dos dispêndios dos diferentes turistas que vão ao evento esportivo, descontando-se os investimentos públicos para se ter uma classificação mais objetiva desta participação em prol da realização do evento esportivo.

Propõe-se, assim, um modelo para se enquadrar três conceitos distintos que contribuem com o processo de tomada de decisão dos diferentes entes públicos em suas respectivas participações e investimentos. Eles estão descritos a seguir:

1) Retorno Direto: compreendido como aquele em que o investimento público direto no evento é menor ou igual ao retorno em tributos proporcionado pelo respectivo evento. É, assim, considerado quando a razão entre as receitas tributárias dividida pelo investimento no evento for igual ou maior do que um.

2) Recirculação Financeira: compreendido como aquele em que o investimento público direto no evento representa um valor menor ou igual ao montante que circulou na localidade a partir do turismo e de investimentos privados. Representa, em outras palavras, um fomento do poder público ao evento, tendo como benefício a movimentação econômica na localidade. Caso o Retorno Direto tenha uma razão menor do que um, faz-se a divisão entre a movimentação econômica do evento pelo investimento público no evento, sendo que o valor deve ser maior ou igual a um para o seu efetivo enquadramento.

3) Impacto Intangível: compreendido como aquele em que o investimento público direto no evento supera o valor de movimentação econômica na localidade. Para estes casos, o ente público precisa pensar estrategicamente o investimento, respeitando-se os objetivos da localidade em expor sua imagem por meio do evento esportivo. A literatura recomenda que o poder público remunere o evento esportivo pelo seu aspecto intangível apenas em casos de eventos classificados como de "Tipo A", compreendidos como aqueles que são de única ocorrência, megaevento e internacional, que proporcionam significativa atividade econômica (GRATTON; DOBSON; SHIBLI, 2000) e, portanto, têm capacidade inconteste de gerar impacto intangível (RODRIGUES, 2016), diferentemente de eventos de menor porte. $O$ teto desta remuneração pelo poder público deve corresponder a, no máximo, 43\% sobre o cálculo feito para a Recirculação Financeira. Este incremento percentual é o equivalente ao valor intangível de aquisição de empresas do setor de mídia e entretenimento (KPMG Europe LLP, 2009), possibilitando, assim, uma analogia de práticas do mercado com o tema em causa, os eventos esportivos. O "Modelo de Impactos Intangíveis de Megaeventos" (ME-I)² proposto por Rodrigues (2016) serve como base de cálculo para o Impacto Intangível, uma vez que reúne 42 indicadores específicos de mensuração de valor, de competitividade e de 
desenvolvimento local de forma sinérgica entre 0 objeto em análise ante as estratégias e expectativas do ente público que investe recursos dos contribuintes.

A Figura 3 ilustra a proposta de análise visando à facilitação e à racionalização da tomada de decisão do poder público no investimento em eventos esportivos. O modelo proposto de valoração impõe, assim, limites sobre os gastos públicos em eventos esportivos, conforme suas características e interesses específicos. A Tabela 4 apresenta os cálculos para se chegar às conclusões da análise.

Figura 3 - Proposta de Análise para Avaliação do Investimento Público em Eventos Esportivos.

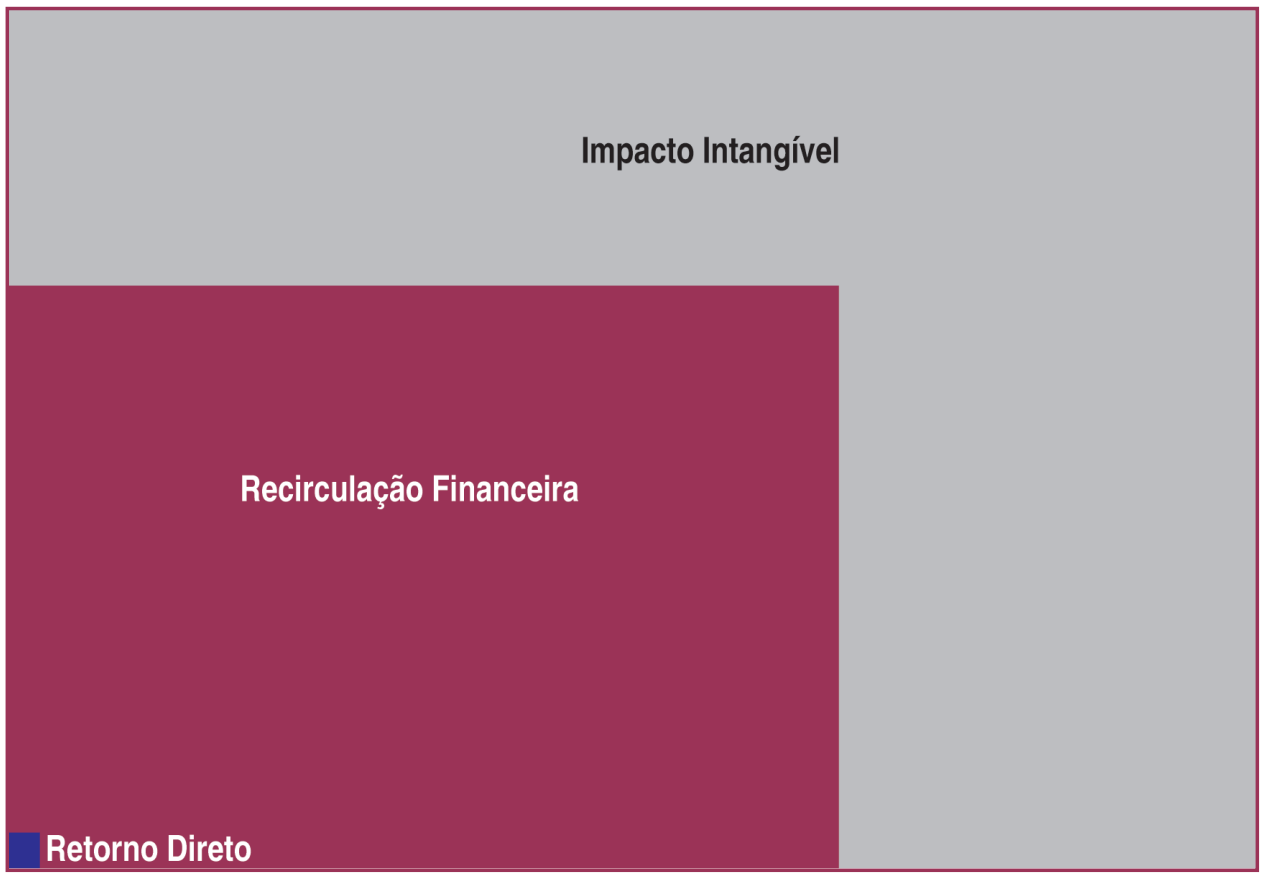

Fonte: dos autores.

Tabela 4 - Proposta de Cálculos para a Simulação da Análise do Investimento Público em um Evento Esportivo.

\begin{tabular}{|c|c|c|}
\hline $\begin{array}{l}\text { PASSO 01: CÁLCULO DO } \\
\text { RETORNO DIRETO }\end{array}$ & $\begin{array}{c}\text { PASSO 02: CÁLCULO DA } \\
\text { RECIRCULAÇÃO FINANCEIRA }\end{array}$ & $\begin{array}{l}\text { PASSO 03: CÁLCULO DO } \\
\text { IMPACTO INTANGÍVEL }\end{array}$ \\
\hline$R D=\frac{R T}{\$}$ & $R F=\frac{I T}{\$}$ & $I I=(R F \times M E I 2 \times(1+c))$ \\
\hline $\begin{array}{l}\text { Se RD >= 1; O EVENTO } \\
\text { ESPORTIVO PROPORCIONA } \\
\text { RECEITA TRIBUTÁRIA PARA } \\
\text { O PODER PÚBLICO APÓS } \\
\text { DESCONTADO O INVESTIMENTO } \\
\text { REALIZADO }\end{array}$ & $\begin{array}{l}\text { Se RF >= 1; O EVENTO } \\
\text { ESPORTIVO APRESENTA } \\
\text { RETORNO DE RECIRCULAÇÃO } \\
\text { FINANCEIRA A PARTIR DO } \\
\text { INVESTIMENTO PÚBLICO }\end{array}$ & $\begin{array}{l}\text { Se RF <= 1; É SINAL DE QUE O } \\
\text { PODER PÚBLICO OPTOU POR } \\
\text { REMUNERAR O EVENTO PELO } \\
\text { SEU IMPACTO INTANGÍVEL E, } \\
\text { PORTANTO, DEVERÁ OBEDECER } \\
\text { A LIMITES DE INVESTIMENTO, } \\
\text { CLASSIFICAÇÃO DO EVENTO E } \\
\text { ESTRATÉGIA }\end{array}$ \\
\hline
\end{tabular}

Fonte: dos autores.

Considerando que:

$\$$ = representa o valor investido pelo poder público em análise no evento esportivo.

$\mathrm{RT}$ = representa a recuperação tributária direta advinda do evento esportivo, correspondente à característica de tributação de cada ente público. 
IT = representa o impacto total, que é dado pela soma do impacto do turismo com a gestão, operação e organização na localidade.

$\mathrm{RD}=$ retorno direto do evento esportivo para o respectivo ente público (municipal, estadual ou federal).

$\mathrm{RF}=$ valor da recirculação financeira do evento esportivo para o respectivo ente público (municipal, estadual ou federal).

II = impacto intangível, para evento de "Tipo A" (GRATTON; DOBSON; SHIBLI, 2000).

MEl2 = score do intangível, dado pela mensuração de valor da metodologia ME-I² (RODRIGUES, 2016), em percentual.

$c=$ fator multiplicador constante, estabelecido em 43\% (KPMG EUROPE LLP, 2009) a partir de ME-I².

Para o X Games, aplicou-se o modelo de análise dos resultados econômicos do evento para cada ente público que investiu na realização do evento, respeitando as características particulares de cada esfera governamental. A análise do Impacto Intangível não foi realizada porque o X Games Brasil se caracteriza como evento de "Tipo C" (GRATTON; DOBSON; SHIBLI, 2000). O resultado é apresentado na Tabela 5.

Tabela 5 - Análise do Impacto Econômico do X Games sobre o Investimento Público de cada Ente Governamental.

\begin{tabular}{|c|c|c|c|c|}
\hline \multicolumn{2}{|c|}{ Análises } & \multirow{2}{*}{$\begin{array}{c}\text { Municipal } \\
0,12\end{array}$} & \multirow{2}{*}{$\begin{array}{c}\text { Estadual } \\
0,81\end{array}$} & \multirow{2}{*}{$\begin{array}{c}\text { Federal } \\
0,74\end{array}$} \\
\hline & Índice & & & \\
\hline Retorno Direto & Resultado & Não houve Retorno Direto & $\begin{array}{l}\text { Não houve Retorno } \\
\text { Direto }\end{array}$ & $\begin{array}{l}\text { Não houve Retorno } \\
\text { Direto }\end{array}$ \\
\hline & Índice & 6,27 & 11,44 & 6,96 \\
\hline Financeira & Resultado & $\begin{array}{l}\text { Houve Recirculação } \\
\text { Financeira }\end{array}$ & $\begin{array}{l}\text { Houve Recirculação } \\
\text { Financeira }\end{array}$ & $\begin{array}{l}\text { Houve Recirculação } \\
\text { Financeira }\end{array}$ \\
\hline
\end{tabular}

Fonte: da pesquisa.

Assim, analisando-se os resultados em prol de cada ente público, tem-se a seguinte avaliação para:

- Prefeitura de FDIPR - para cada R\$1,00 investido pela prefeitura no X Games, houve recuperação tributária de $\mathrm{R} \$ 0,12$, sem proporcionar Retorno Direto; mas o investimento se justificou, pois houve Recirculação Financeira, com índice de $R$ \$ 6,27 para cada $R \$ 1,00$ investido;

- Governo do Estado do Paraná - para cada R\$1,00 investido pelo Estado, houve uma recuperação tributária de $\mathrm{R} \$ 0,81$, sem proporcionar Retorno Direto; mas o investimento foi amparado pela movimentação econômica de $R$ \$ 11,44 para cada $R$ \$ 1,00 investido, representando uma "Recirculação Financeira" em prol dos habitantes do Estado;

- Governo Federal-cada R \$1,00 investido pelo Governo Federal gerou recuperação tributária de R 0,74, sem proporcionar Retorno Direto; mas com uma movimentação econômica de $\mathrm{R} \$ 6,96$ para cada $\mathrm{R} \$ 1,00$ investido, representando uma "Recirculação Financeira" em prol da população nacional.

Esses índices indicam que a "balança comercial" do evento em análise foi negativa, com importante aporte público para o equilíbrio econômico-financeiro do X Games em FDIPR. A tomada de decisão pelos investimentos pautada na "Recirculação Financeira" é plausível na medida em que estimula o esporte (HEINEMANN, 2001) e, ao mesmo tempo, gera novos negócios em prol do comércio, indústria e serviços da localidade. 
A proposta de análise se converte em subsídio para novos estudos, uma vez que precisará ser amplamente testada e aplicada para que embase as decisões de investimento antes da realização dos eventos, garantindo confiabilidade e segurança para que o ente público aplique recursos de modo mais racional no esporte.

\section{CONCLUSÕES}

Para a sustentabilidade do movimento esportivo, é fundamental constituir um processo de racionalização dos investimentos em grandes projetos. Propõe-se, assim, uma conta mais verdadeira e racional, que facilite as tomadas de decisão do poder público no apoio à realização de um evento esportivo em sua região de abrangência. Ao aplicar uma metodologia com este viés no Brasil, o país se aproxima de casos bem-sucedidos de países como Reino Unido, Canadá e Estados Unidos da América, que utilizam processos próprios e adequados às suas características específicas, para subsidiar a participação pública em investimentos em prol do esporte, com ferramentas como o Event/mpact (UK SPORTS, 2016); ; o Sport Tourism Economic Assessment Model (STEAM, 2016) ${ }^{4}$ e o Economic Impact Modeling (IMPLAN, 2016), respectivamente. ${ }^{5}$

A concepção de um modelo mais racional de análise do impacto econômico no Brasil se torna relevante também aos proponentes de eventos esportivos, que comumente se dedicam à entrega de valor para patrocinadores, competidores e público em geral, mas ignoram ou negligenciam as contrapartidas em favor dos entes públicos.

A proposição deste método de análise deve facilitar o planejamento e a posterior gestão e operação de eventos esportivos, de modo a torná-lo sustentável economicamente a partir dos investimentos públicos, minimizando a opinião de movimentos contrários. A construção de um ciclo virtuoso de investimentos que possa corroborar o aporte público ou parcerias públicoprivadas para a realização de eventos, de modo a ser benéfico para todos os envolvidos, é fundamental para esse processo.

\section{REFERÊNCIAS}

ACCESS ECONOMIC PTY LIMITED. Cost benefit analysis of the 2022 FIFA World Cup. Canberra: Department of Resources, Energy and Tourism, 2010.

BARGET, Eric; FERRAND, Alain. Impact économique des événements sportifs sur le territoire: une méthode d'analyse basée sur les échanges entre les parties prenantes. Revue Management \& Avenir, n. 57, p. 96-112, 2012.

BARGET, Eric; GOUGOUT, Jean-Jacques. Hosting mega-sporting events: which decision-making rule? International Journal of Sport Finance, n. 5, p.141-162, 2010.

BERNTHAL, Matthew J.; REGAN, Thomas $\mathrm{H}$. The economic impact of NASCAR racetrack on a rural community and region. Sport Marketing Quarterly, v.13, n.1, p. 26-34, 2004.

3 UK SPORTS. EVENT IMPACT, 2016. Disponivel em: <http://www.eventimpacts.com/>. Acesso em: 25 out. 2016. 
BRASIL. MINISTÉRIO DO TURISMO. Anuário Estatístico de Turismo: Ano Base: 2013. Brasília : Secretaria Nacional de Políticas de Turismo, 2014.

BRUNET, Ferran; XINWEN, Zuo. The economy of the Beijing Olympic Games: an analysis of first impacts and prospects. Centre d'Estudis Olímpics UAB. Barcelona, 2009. Disponível em: <http:// olympicstudies.uab.es/pdf/wp116 eng.pdf>. Acesso em: 25 out. 2016.

CROMPTON, John L. Economic impact analysis of sports facilities and events: eleven sources of misapplication. Journal of Sport Management, v. 9, n.1, p.14-35, 1995.

CROMPTON, John L. The economic sports and events impact of tournaments. Parks \& Recreation, v.34, n.9, p. 142-150, 1999.

DANIELS, Margaret J.; NORMAN, William C. Estimating the economic impact of seven regular sport tourism events. Journal of Sport \& Tourism, v.8,n.4, p. 214-222, 2003.

EDWARDS, Allan; SKINNER, James. Qualitative research in sport management. Oxford: Elsevier, 2009 .

FOZ DO IGUAÇU. SECRETARIA MUNICIPAL DE TURISMO DE. Inventário da Oferta Turística de Foz do Iguaçu. Foz do Iguaçu, 2014. p. 210.

FRAWLEY, Stephen; CUSH, Adam. Major sport events and participation legacy: the case of the 2003 Rugby World Cup. Managing Leisure, n. 16, p. 65-76, 2011.

GIL, Antônio Carlos. Como elaborar projetos de pesquisa. São Paulo: Atlas, 2010.

GRATTON, Chris; DOBSON, Nigel; SHIBLI, Simon. The economic importance of major sports events: a case-study of six events. Managing Leisure, v.5, n.1, p. 17-28, 2000.

GRATTON, Chris; PREUSS, Holger. Maximizing Olympic impacts by building up legacies. The International Journal of the History of Sport, v.25, n. 14, p. 1922-1938, Dec. 2008.

GRATTON, Chris; SHIBLI, Shibli; COLEMAN, Richard. The economic impact of major sports events: a review of ten events in the UK. Sociological Review,v.54, n.S2, p. 41-58, Dec. 2006.

HEINEMANN, Klaus. La repercusión económica del deporte: marco teórico y problemas prácticos. EFDeportes.com, n. 43, dec. 2001. Disponível em: <http://www.efdeportes.com/efd43/econom.htm>. Acesso em: 25 out. 2016.

HUANG, Haiyan; HANQI, Gao. Estimation of the non-market value generated by 2009 Shanghai ATP Masters 1000: a case study of the value of civic pride. Advances in Applied Economics, Business and Development, v. 209, p.549-554, 2011.

JEANRENAUD, Claude. Sports events: uses and abuses of economic impact studies. Finance \& The Common Good, n. 26, p. 99-104, 2006.

KASIMATI, Evangelia. Economic aspects and the Summer Olympics: a review of related research. International Journal of Tourism Research, n. 5, p. 433-444, 2003.

KAVETSOS, Georgios; SZYMANSKI, Stefan. From the Olympics to the grassroots: what will London 2012 mean for sport funding and participation in Britain. Public Policy Research, v.16, n.3, p. 192196, 2009. 
KPMG EUROPE LLP. Intangible assets and goodwill in the context of business combinations: an industry study. Corporate Finance. Munique, 2009. 89p. Disponível em: <http://www.kpmg.com/PT/pt/ IssuesAndlnsights/Documents/Intangible-assets-and-goodwill.pdf>. Acesso em: 25 out. 2016.

LEE, Cheng-Jong. Effects of sport mega-events on city brand awareness and image: using the 2009 world games in Kaohsiung as an example. Quality and quantity, v.48, n.3, p.1243-1256, 2014.

OBSERVATÓRIO DE TURISMO DE FOZ DO IGUAÇU. Perfil do Hóspede de Foz do Iguaçu. Foz do Iguaçu, 2014a.

OBSERVATÓRIO DE TURISMO DE FOZ DO IGUAÇU. Pesquisa de Ocupação Hoteleira realizada pela ABIH e SINDHOTÉIS de Foz do Iguaçu. Foz do Iguaçu, 2014b.

OBSERVATÓRIO DE TURISMO DE FOZ DO IGUAÇU. Comparativo da Oferta Turística 1987 a 2014. Foz do Iguaçu, 2014c.

OBSERVATÓRIO DO TURISMO DA CIDADE DE SÃO PAULO. São Paulo Indy 300 - Perfil de Público. São Paulo, 2012.

PORTER, Philip K.; FLETCHER, Deborah. The economic impact of the Olympic Games: ex ante predictions and ex post reality. Journal of Sport Management, v.22, n.4, p. 470-486, 2008.

PREUSS, Holger; SCHÜTTE, Norbert; KÖNECKE, Thomas; DACOSTA, Lamartine. Olympic ideals as seen by Olympic scholars and experts. Mainz: University Mainz Johannes Gutenberg, 2014. (Working Paper Series, 13).

RAMCHANDANI, Girish M.; COLEMAN, Richard J. Testing the accuracy of event economic impact forecasts. International Journal of Event and Festival Management, v.3, n. 2, p.188-200, 2012.

RODRIGUES, Mauricio N. Impact/Legacy measurement and evaluation in Mega Event projects with focus on intangible assets. 2006. $245 \mathrm{f}$. Tese (Doutorado em Ciências da Gestão) - Instituto Alberto Luiz Coimbra de Pós-Graduação e Pesquisa de Engenharia, Universidade Federal do Rio de Janeiro, Rio de Janeiro. 2016.

SACK, Allen L.; JOHNSON, Arthur T. Politics, economic development, and the Volvo International Tennis Tournament. Journal of Sport Management, v.10, n.1, p.1-14, 1996.

SILVESTRE, Gabriel. The social impacts of mega-events: towards a framework. Revista Esporte e Sociedade, v.4, n.10, p. 1-26, nov. 2008/fev. 2009.

TAKS, Marijke; KESENNE, Stefan; CHALIP, Laurence; GREEN, Christine B. Economic impact analysis versus cost benefit analysis: the case of a medium-sized sport event. International Journal of Sport Finance, n. 6, 187-203, 2011.

TIEN, Chengli; LO, Huai-Chun.; LIN, Hsiou-Wei. The economic benefits of mega events: a myth or a reality? A longitudinal study on the Olympic Games. Journal of Sport Management, v. 25, p.11-23, 2011.

WALO, Maree; BULL, Adrian; BREEN, Helen. Achieving economic benefit at local events: a case study of a local sports event. Journal of Festival Management and Event Tourism, v.4, p.95-106, 1996.

WEINSTEIN, Roy; DEGRACIA, Jeremy; LIN, Edna. Economic Impact of the 2011 NBA All-Star Game on Los Angeles County. Los Angeles: NBA, 2010. p. 12. 
WICKER, Pamela; HALLMANN, Kirstin; BREUER, Christoph; FEILER, Svenja et al. The value of Olympic success and the intangible effects of sport events: a contingent valuation approach in Germany. European Sport Management Quarterly, n. 12, n.4, p. 337-355, 2012.

WILSON, Robert. The economic impact of local sport events: significant, limited or otherwise? A case study of four swimming events. Managing Leisure, v.11, n.1, p. 57-70, 2006.

YU, Yu; LIU, Jiangyong. A comparative analysis of the Olympic impact in East Asia: from Japan, South Korea to China. The International Journal of the History of Sport, v.28, n. 16, p. 2290-2308, 2011. 
